\title{
Missed curable carcinoma of the pancreas presenting as chronic pancreatitis
}

\author{
SM Wharton, Z Rahman, CD Johnson
}

\begin{abstract}
Summary
We present a case of pancreatic malignancy which presented as chronic pancreatitis. The diagnostic difficulties are discussed. We recommend that when there is any doubt about a diagnosis of chronic pancreatitis with a pancreatic mass, then early resection is appropriate.
\end{abstract}

Keywords: pancreatic cancer, chronic pancreatitis, diagnosis

It has always been a notoriously difficult diagnostic problem to distinguish between chronic pancreatitis and carcinoma of the pancreas. These conditions may coexist in the same patient ${ }^{1}$ and it is often impossible to determine which came first. Chronic pancreatitis and pancreatic carcinoma also share many clinical features including upper abdominal pain, obstructive jaundice, anorexia, weight loss, steatorrhoea and diabetes mellitus. ${ }^{2}$ Consequently, even when a firm diagnosis of chronic pancreatitis is made, there is often doubt that pancreatic carcinoma may also be present.

There have been many studies of the diagnostic criteria for these conditions, ${ }^{1-4}$ which attempt to produce a reliable investigative protocol to aid in diagnosis. Because of the diagnostic difficulty, some surgeons have advocated a policy of radical resection when any possibility exists that carcinoma may be present in addition to chronic pancreatitis. The lesson from the following case presentation is that failure to follow this recommendation may delay diagnosis until the tumour is incurable.

\section{Case report}

A 53-year-old woman presented with obstructive jaundice to another hospital in early 1992 . There was no history of high alcohol intake or other predisposing factors. Endoscopic retrograde cholangiopancreatography (ERCP) showed a possible cholangiocarcinoma and, although computed tomography (CT) showed distal common bile duct obstruction (figure 1A), no mass was seen. At laparotomy, the whole pancreas was found to be inflamed and hard with no obvious mass in the head of the gland (figure 1B). Therefore, a chloledochojejunostomy was performed to alleviate the jaundice. Biopsies at operation showed changes consistent with chronic pancreatitis.

The patient returned in January 1993 complaining of abdominal pain. CT scan at this time showed a 4-cm pancreatic head mass with an atrophic tail and dilated pancreatic duct. Repeated CT scan three months later showed no further change.

In August 1993 the patient returned complaining of anorexia, abdominal pain and ascites. She was referred to our unit where a further CT scan showed little change from the previous one (figure 2). Repeated cytology of the ascitic fluid and biopsy of the pancreas showed no evidence of malignancy, although

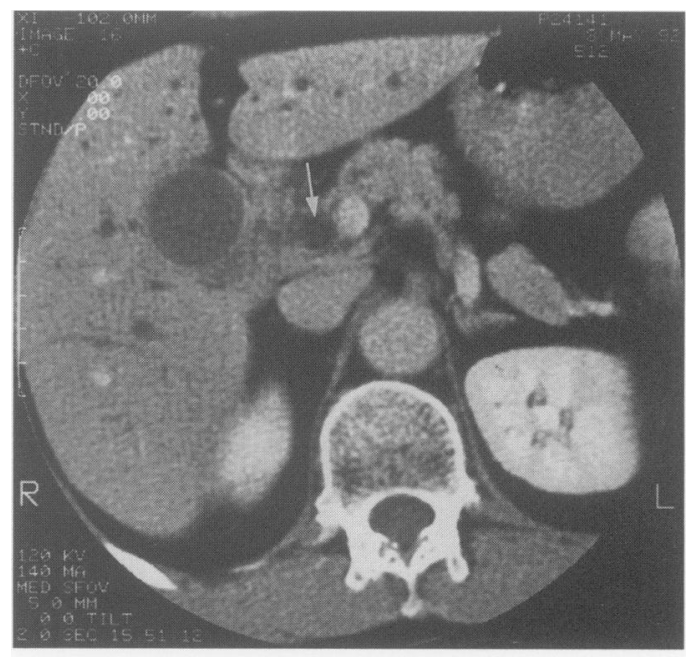

A

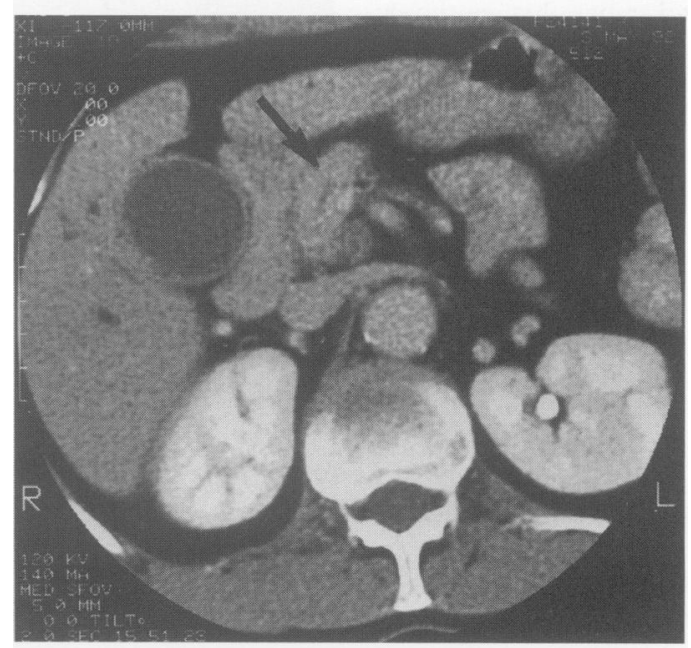

B

Figure 1 CT scan on presentation. (A) Dilated intrahepatic bile ducts and distended gall bladder; the common hepatic duct (white arrow) lies adjacent to the enhanced portal vein, and is distended; (B) the pancreatic head (black arrow) appears normal 
one fine-needle aspiration from the head of the pancreas was reported to be suggestive of chronic pancreatitis, but 'suspicious of malignancy'. Subsequently, a 3-cm mass appeared in the left supraclavicular fossa. Aspiration of this mass yielded clear fluid; cytology was negative. The mass disappeared after aspiration, but recurred and was excised in January 1994. Histology showed cystic areas lined by cuboidal and glandular epithelium (figures 3 and 4). This was suggestive of a metastasis from a pancreatic cystadenocarcinoma. The ascites was drained by the insertion of a
Figure 2 CT scan seven months after operation. There is a $4-\mathrm{cm}$ mass replacing the pancreatic head (white arrow) and some surrounding infiltrative changes

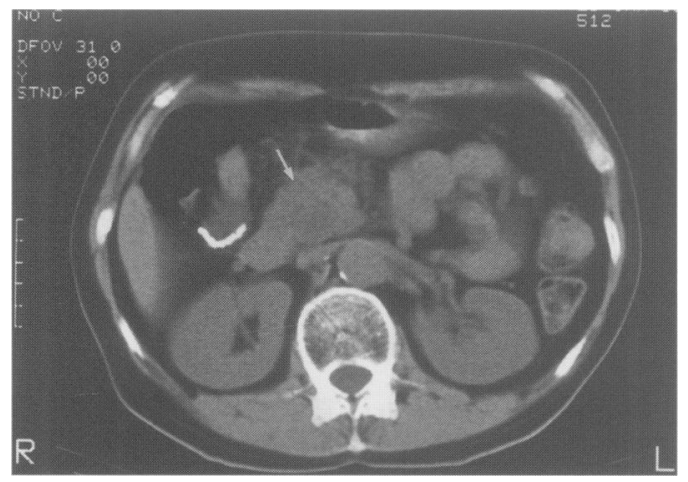

Figure 3 Well-differentiated glandular tumour has glands of varying sizes separated by a lymphocyte rich stoma. $\mathrm{H} \& \mathrm{E} \times 100$ original
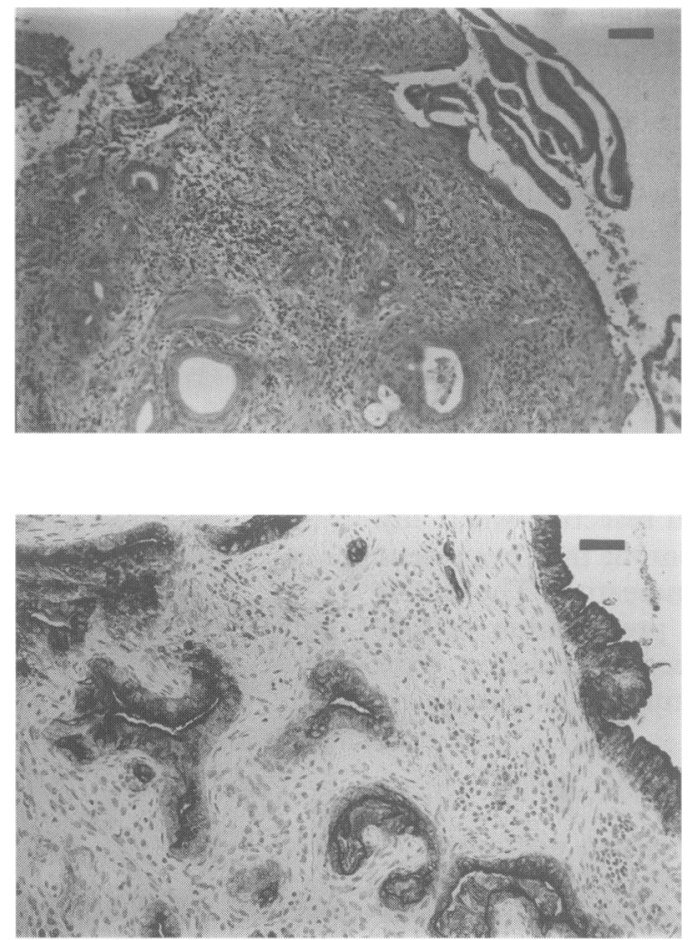

Figure 4 Immunocytochemical staining for cytokeratins has positively stained the epithelial component of the tumour. CAM $5.2 \times 250$ original peritoneovenous shunt. In May 1994 the patient was anaemic and weak, but with no evidence of further metastases. She died in November 1994.

\section{Discussion}

Since chronic pancreatitis and carcinoma of the pancreas may coexist in the same patient, it can be difficult or impossible to detect carcinoma in a patient presenting with apparent chronic pancreatitis. Also, the symptoms and signs of pancreatitis and carcinoma are not specific. Until recently, the aetiological relationship between these two conditions has not been clear. ${ }^{5}$ Risk factors for chronic pancreatitis such as high alcohol intake are possible, but not confirmed, risk factors for pancreatic carcinoma. ${ }^{2}$ White and $\mathrm{Hart}^{6}$ found that $20 \%$ of patients known to have pancreatitis on the basis of alcoholism were found at operation to have cancer. It is now established that chronic pancreatitis is a predisposing condition for pancreatic cancer. ${ }^{7,8}$ However, in this case the initial presentation can be explained on the basis of a small tumour causing obstructive pancreatitis, as there is little evidence of prior symptoms of chronic pancreatitis, and the interval between the first and second operations was relatively short.

Given the poor prognosis of pancreatic carcinoma which results from its usual late presentation and with the recent reduction in the operative risk of pancreatic surgery, it has been argued that resection should be performed early, even where the diagnosis of cancer is suspected but not confirmed. Carter ${ }^{2}$ argued that occult cancer in a gland with pancreatitis may be more amenable to resection and its seems probable that such early tumours are likely to have a better prognosis. Diagnosis depends upon a range of investigations (table) none of which is particularly sensitive in distinguishing between chronic pancreatitis and pancreatic carcinoma.

White and $\mathrm{Hart}^{6}$ found that abdominal ultrasonography was the most useful imaging technique in the diagnosis of pancreatitis. Hunt and Blumgart ${ }^{4}$ found pancreatic juice cytology to be a highly specific way of diagnosing carcinoma in the presence of pancreatitis. However, they found a high degree of false-negative results which limits its value in making this important discrimination. In the case presented here, pancreatic cytology was not performed at the initial ERCP. A positive result would have been of diagnostic value, but a negative result would have been unhelpful.

The problem of a high false-negative rate also besets intra-operative and CT-guided biopsy. This is because the inflammatory reaction around a small tumour may be extensive. ${ }^{1}$ This is demonstrated well in this case where repeated biopsies were either inconclusive or showed only inflammatory change. Even when the correct site is found, it is often difficult for the histopathologist to distinguish between inflammatory change and well-differentiated adenocarcinoma, ${ }^{2}$ although

52

86

$89-90$

86

85
Cytology and fine-needle aspiration Pancreatic onocofetal antigen
History and clinical examination

$50-90$

$-$ 
diagnosis is aided by the use of immunocytochemistry.

Tumour markers are gaining popularity in the diagnosis and monitoring of malignant disease. In pancreatic carcinoma three markers have commanded most interest: carcinoembryonic antigen (CEA), alpha-fetoprotein and pancreatic oncofetal antigen (POA). CEA is commonly high in patients with pancreatic carcinoma, but is also high in patients with other malignancies and with benign disease. ${ }^{3}$ Histologically, tumour cells stain positive for CEA, but so may non-tumour cells. ${ }^{2}$ Only when the tumour is advanced and inoperable is CEA raised to a high level and even then it is not specific to tumours of pancreatic origin. ${ }^{3}$ High alpha-fetoprotein levels appear to be even less specific to pancreatic carcinoma. POA is similarly non-specific to pancreatic cancer, although it may have some value in the monitoring of patients following resection of a POA-secreting tumour. ${ }^{3}$ Carter $^{2}$ found the marker CA 19-9 disappointing as a possible marker for early pancreatic carcinoma and the specificity in distinguishing pancreatitis from carcinoma was poor.

The mortality of pancreatic resection surgery is declining. ${ }^{9}$ Most series now report a hospital mortality well under $10 \%$, and a review of publications in the last 10 years shows a median postoperative mortality below $5 \% .^{10}$ Data on whether patients with early pancreatic carcinoma survive for longer if resection is performed early is inconclusive. However, if the tumour is confined to the pancreas, and all resection margins are clear, survival after five years is about $25 \% .^{9}$ Therefore, resection appears justified, particularly for patients with

1 Becker V. Carcinoma of the pancreas and chronic pancreatitis - a possible relationship. Acta Hepatico Gastroentero 978; 25: 257-9.

2 Carter DC. Cancer of the head of the pancreas or chronic pancreatitis? A diagnostic dilemma. Surgery 1992; 111 602-3.

3 Moosa AR, Levin B. The diagnosis of "early" pancreatic cancer: the University of Chicago experience. Cancer 1981; 47: $1688-97$

4 Hunt DR, Blumgart LH. Pre-operative differentiation between carcinoma of the pancreas and chronic pancreatitis: between carcinoma of the pancreas and chronic pancreatitis:

5 the contribution of cytology. Endoscopy 1982; 14: 171-3. Haddock G, Carter DC. Ae

6 White TT, Hart MJ. Pancreaticojejunostomy versus resection in the treatment of chronic pancreatitis. Am $\mathcal{F}$ Surg 1979; 138: $129-33$.

7 Ramesh H, Augustine P. Surgery and tropical pancreatitis: analysis and risk factors. Br f Surg 1992; 79: 544-9.

8 Lowenfels AB, Maisonneuve EV, Cavallini G, et al and the International Pancreatitis Study Group. Pancreatitis and the risk of pancreatic cancer. N Engl F Med 1993; 328:1433-7.

\section{Learning point}

When there is any possibility of the existence of carcinoma in the presence of chronic pancreatitis, then the policy should be for early radical resection

small tumours in whom diagnostic certainty may be hard to achieve. Only a policy of resection even when confirmation of the diagnosis is lacking, will allow some of these curative resections. Smith et $a l^{11}$ found that operative resection for carcinoma based on clinical presentation, laboratory results, and diagnostic imaging produced misdiagnosis in only $5 \%$ of cases. However, no mention was made of the percentage of patients not operated on, who subsequently turned out to have a malignancy, as in the case presented here. This case clearly illustrates the lost opportunity for curative resection at the time of the initial presentation, and the continuing diagnostic difficulty which persisted until a metastasis was removed from the supraclavicular fossa. This is particularly disappointing in this case as resection of localised cystadenocarcinoma is usually curative. ${ }^{12}$ These less-aggressive slow-growing tumours may well cause more diagnostic confusion than the more usual ductal adenocarcinoma. We therefore agree with Carter $^{2}$ that, in the absence of a firm diagnosis of carcinoma, the surgeon should "regard the Whipple operation as the most effective and reliable method of providing the pathologist with a biopsy of the head of the pancreas".

9 Trede M. Survival after pancreato-duodenectomy. 118 consecutive resections without an operative mortality. Ann Surg 1990; 211: 447-58.

10 Glazer G, Coulter C, Crofton ME, et al. Controversial issues in the management of pancreatic cancer: part two. Ann R Coll Surg Engl 1995; 77: 174-80.

11 Smith CD, Behrns KE, van Heerden JA, Sarr MG. Radical pancreatoduodenectomy for misdiagnosed pancreatic mass. Br ₹ Surg 1994; 81: $585-9$.

12 Capangno J, Oertel JE. Mucinous cystic neoplasms of the pancreas with overt and latent malignancy (cystadenocarcinoma and cystadenoma): a clinicopathological study of 41 cases. Am f Clin Pathol 1978; 69: 573-80.

13 Freeny PC. Imaging in chronic pancreatitis: a synopsis. In: Beger HG, Bucher M, Ditschuneit H, Malfertheiner P, eds. Chronic pancreatitis. Berlin: Springer-Verlag, 1990; pp 33041 